\title{
Criminologie
}

\section{Analyse de la structure sociale des conflits et des alliances intergangs}

\section{Karine Descormiers et Carlo Morselli}

Volume 43, numéro 1, printemps-été 2010

Les jeunes et la rue

URI : https://id.erudit.org/iderudit/044051ar

DOI : https://doi.org/10.7202/044051ar

Aller au sommaire du numéro

\section{Éditeur(s)}

Les Presses de l'Université de Montréal

ISSN

0316-0041 (imprimé)

1492-1367 (numérique)

Découvrir la revue

Citer cet article

Descormiers, K. \& Morselli, C. (2010). Analyse de la structure sociale des conflits et des alliances intergangs. Criminologie, 43(1), 57-89.

https://doi.org/10.7202/044051ar
Résumé de l'article

Cette étude propose un cadre analytique qui permet d'étudier le réseau social des gangs montréalais, dont certains s'identifient aux deux grandes coalitions étatsuniennes : les Bloods (les rouges) et les Crips (les bleus). L'objectif de cette recherche est de décrire la structure de ce réseau social en s'intéressant d'abord à la constitution et à l'organisation interne de ces gangs et, ensuite, aux dynamiques relationnelles qui les unissent une fois mis en interaction les uns avec les autres. Nous souhaitons donc comprendre dans quelle mesure les dynamiques intergangs, tant positives que négatives, s'orchestrent selon la conception polarisée des conflits Crips versus Bloods. Nos données ont été recueillies auprès de 20 membres juvéniles de gangs pris en charge par le Centre jeunesse de Montréal-Institut universitaire. Ces participants, qui appartiennent à différents gangs rivaux ou alliés, ont été rencontrés dans le cadre d'une série d'entrevues de groupe réalisée au cours de l'année 2007. Ensemble, ils ont identifié un total de 35 gangs qui occupent des territoires précis à Montréal. La structure obtenue comprend des modèles de relations attendus par la conception traditionnelle, conflits inter-consortiums (77 \% de l'ensemble des dynamiques négatives $n=43$ ) et alliances intra-consortiums ( $87 \%$ de l'ensemble des dynamiques positives $n=40$ ). Des exceptions à cette conception sont soulevées et nuancent la compréhension de la structure relationnelle à l'étude.
Tous droits réservés @ Les Presses de l’Université de Montréal, 2010
Ce document est protégé par la loi sur le droit d'auteur. L’utilisation des services d'Érudit (y compris la reproduction) est assujettie à sa politique d'utilisation que vous pouvez consulter en ligne.

https://apropos.erudit.org/fr/usagers/politique-dutilisation/ 


\title{
Analyse de la structure sociale des conflits et des alliances intergangs
}

\author{
Karine Descormiers \\ Étudiante au doctorat \\ Département de criminologie, Simon Fraser University \\ Karine_Descormiers@sfu.ca \\ Carlo Morselli \\ Professeur agrégé \\ École de criminologie, Université de Montréal \\ carlo.morselli@umontreal.ca
}

RÉSUMÉ - Cette étude propose un cadre analytique qui permet d'étudier le réseau social des gangs montréalais, dont certains s'identifient aux deux grandes coalitions étatsuniennes: les Bloods (les rouges) et les Crips (les bleus). L'objectif de cette recherche est de décrire la structure de ce réseau social en s'intéressant d'abord à la constitution et à l'organisation interne de ces gangs et, ensuite, aux dynamiques relationnelles qui les unissent une fois mis en interaction les uns avec les autres. Nous souhaitons donc comprendre dans quelle mesure les dynamiques intergangs, tant positives que négatives, s'orchestrent selon la conception polarisée des conflits Crips versus Bloods. Nos données ont été recueillies auprès de 20 membres juvéniles de gangs pris en charge par le Centre jeunesse de Montréal-Institut universitaire. Ces participants, qui appartiennent à différents gangs rivaux ou alliés, ont été rencontrés dans le cadre d'une série d'entrevues de groupe réalisée au cours de l'année 2007. Ensemble, ils ont identifié un total de 35 gangs qui occupent des territoires précis à Montréal. La structure obtenue comprend des modèles de relations attendus par la conception traditionnelle, conflits inter-consortiums ( $77 \%$ de l'ensemble des dynamiques négatives $n=43$ ) et alliances intra-consortiums ( $87 \%$ de l'ensemble des dynamiques positives $n=40$ ). Des exceptions à cette conception sont soulevées et nuancent la compréhension de la structure relationnelle à l'étude.

MOTS CLÉS - Gangs, Crips, Bloods, structure interne, réseau social, alliances et rivalités intergangs. 


\section{Introduction ${ }^{1}$}

Montréal, zone urbaine où la présence des gangs de rue est manifeste. En 2007, le Service de police de la Ville de Montréal dénombre une vingtaine de gangs majeurs tapissant le territoire montréalais. En 2002, différentes agences de police canadiennes estiment que la moyenne d'âge des (approximativement) 7000 membres de gangs au pays est en dessous de 21 ans (Chettleburgh, 2007). D'hier à aujourd'hui, ces regroupements et la population générale des gangs sont systématiquement catégorisés sous l'ombrelle d'une des deux grandes coalitions étatsuniennes: les Bloods (les rouges) et les Crips (les bleus). L'accent mis sur cette dichotomie par les services policiers et les divers médias a créé une représentation polarisée des gangs ainsi que du climat de tension intergang sur la scène montréalaise (Morselli, 2009). Comme d'autres, les gangs montréalais chériraient des couleurs similaires aux grandes coalitions originaires des villes des États-Unis, sans pour autant avoir de réelles affiliations nationales (Starbuck et al., 2001). Puisque l'inspiration d'une telle conception provient d'un contexte étatsunien et date des années 1970, nous ne pouvons pas présumer une transposition intégrale des dynamiques relationnelles ainsi qu'un mimétisme (Felson, 2006). À ce jour, peu d'études scientifiques canadiennes ont bénéficié d'un design de recherche permettant de décrire précisément les réelles couleurs des gangs montréalais et encore moins la nature de leurs interactions.

Cette étude propose un cadre analytique qui permet d'étudier le réseau social des gangs montréalais, dont certains s'identifient aux deux grandes coalitions étatsuniennes: les Bloods et les Crips. L'objectif de cette étude est de décrire la structure de ce réseau social en s'intéressant d'abord à la constitution et à l'organisation interne de ces gangs et ensuite, aux dynamiques relationnelles qui les unissent une fois mis en interactions les uns avec les autres. Nous souhaitons donc comprendre dans quelle mesure les dynamiques intergangs, tant positives que négatives, s'orchestrent selon la conception polarisée des conflits Crips versus Bloods. Au-delà du processus de classification et d'étiquetage des gangs,

1. Cette étude n'aurait été possible sans la grande collaboration du Centre jeunesse de Montréal-Institut universitaire. Nous tenons à remercier spécialement Clément Laporte du Centre d'expertise sur la délinquance des jeunes et les difficultés de comportement qui nous a appuyés dans l'élaboration et la réalisation de ce projet. Merci également à Martin Bouchard pour ses commentaires à diverses étapes de la rédaction de cet article, aux évaluateurs anonymes ainsi qu'à Liette Dion. 
dans quelle mesure leur structure relationnelle s'organise-t-elle autour de la conception de rivalités inter-consortiums? À l'inverse, les alliances intergangs seraient-elles possibles qu'entre entités d'un même consortium (par exemple, uniquement entre gangs Crips) ? L'étude a été réalisée grâce à la participation de 20 membres de gangs détenus dans les établissements du Centre jeunesse de Montréal-Institut universitaire.

\section{Groupes sociaux, dynamiques et conceptualisation}

Selon Fleisher (2005), les gangs de rue seraient considérés comme des groupes sociaux où les membres sont unis les uns aux autres par l'appartenance à un gang d'allégeance spécifique, ce qui résulte en une identité collective commune qui les distingue des membres des autres gangs. La différence d'allégeance, l'animosité et le conflit deviennent des catalyseurs des processus collectifs, justifient l'existence même du gang et consolident la cohésion du groupe (entre autres Thrasher, 1927; Klein, 1971; et Sanchez-Jankowski, 1991, y font référence). Les dynamiques conflictuelles antagoniques se retrouvent au cœur même de l'histoire et, encore à ce jour, au sein de la culture sous-jacente au phénomène des gangs. Si nous nous référons à l'histoire des quatre «super-sized gangs», nous constatons qu'à Chicago les gangs affiliés aux People s'opposent depuis toujours aux gangs affiliés aux Folks et viceversa, alors qu'à Los Angeles, les gangs Crips entretiennent des relations négatives avec les Bloods, d'ailleurs créés en riposte aux attaques des Crips dans les années 1960 (Grennan et al., 2000; Delaney, 2006). Depuis, les relations intergangs antagoniques tendent à être catégorisées dans ce cadre conceptuel. D’ailleurs, les différentes agences d'application de la loi prioriseraient cette vision, c'est-à-dire de regroupements largement organisés où la violence s'actualiserait exclusivement entre les consortiums rivaux plutôt qu'entre les membres d'une même bannière (Jackson et McBride, 1985; Conly, 1993).

Les recherches passées ont composé avec ce précepte, soit une classification prédéfinie qui s'inspire des grandes rivalités populaires interconsortiums (entre autres Decker et Van Winkle, 1994; Fleisher, 1995; Decker, 1996; Short, 1996; Maxson, 1998; Decker et Curry, 2002; McGloin, 2005 ; Short Jr. et Hughes, 2006). Les échantillons de ces recherches étaient composés de membres de gangs qui s'identifiaient à un consortium particulier (Crips, Bloods) ou une nation (People, Folks), pour suivre la terminologie exacte de ces coalitions. Les dynamiques 
antagoniques, particulièrement les homicides, ont été abordées par ces auteurs, et plus indirectement par d'autres. Le Boston Gun Project est l'un des projets de recherche les plus ambitieux en ce qui concerne l'étude de la structure sociale des homicides perpétrés par les gangs; Kennedy, Braga et Piehl (1997) ont eu recours à des entrevues de groupe avec différents acteurs engagés dans la lutte aux gangs (officiers de police, agents de probation et travailleurs sociaux) pour cartographier le territoire de façon à cibler les gangs centraux et les interactions intergangs. Ce projet a été conduit spécifiquement pour élaborer des stratégies d'intervention dans le but de contrer les vagues de violence dans la ville. Ses conclusions demeurent des indicateurs quant aux gangs à cibler pour réduire, voire enrayer, la violence perpétrée sur leur territoire. Leur étude a inspiré d'autres chercheurs qui ont appliqué la perspective des réseaux sociaux aux études des dynamiques de violence intergangs. Par exemple, McGloin (2005) a mené une étude en collaboration avec le New Jersey Gang Task Force, à Newark. Une série de 32 entrevues de groupe avec des experts policiers a permis d'identifier les membres de gangs et leurs associés affiliés à quatre des consortiums dominant le territoire étudié, soit Bloods, Crips, Latin Kings et Netas. On se serait attendu à obtenir un réseau social concentré à certains endroits où il $\mathrm{y}$ a beaucoup de violence (hot spots). Cependant, la structure sociale trouvée est constituée d'entités plutôt dispersées sur le territoire. La structure s'organiserait davantage autour de certains sous-groupes cohésifs qu'autour d'un seul réseau central. Ce résultat reflèterait le laxisme de la structure sociale documenté dans les écrits sur l'organisation sociale des gangs (Yablonsky, 1962; Decker et Van Winkle, 1996; Decker et al., 1998; Howell et al., 2002). Les résultats des travaux de Kennedy et al. (1997) et Mc Gloin (2005) concernent davantage la représentation spatiale du réseau et ses caractéristiques organisationnelles. Les résultats ne s'intéressent pas à l'identification des entités et des consortiums en jeu. Il est sous-entendu que les dynamiques conflictuelles étudiées vont dans le sens prescrit par la conception d'antagonistes intergangs.

Les travaux de Papachristos (2009) et Decker et Curry (2002) poussent l'étude des structures relationnelles à un autre niveau, en précisant les entités concernées dans les conflits. Papachristos (2009) s'est penché sur la structure sociale des homicides liés aux gangs de Chicago, dans les années 1994, 1998 et 2002. Au-delà de la description de la structure relationnelle des gangs étudiés, l'auteur présente deux 
modèles distincts d'interaction: des conflits intra-raciaux et des conflits intra-gangs. Premièrement, les conflits intra-raciaux sont le type d'interaction le plus documentés lorsqu'il est question de la violence attribuée aux gangs. En ce sens, seulement 3\% des interactions de l'échantillon de Papachristos sont des conflits entre gangs qui n'appartiennent pas au même groupe ethnique. Les homicides perpétrés par les gangs concerneraient, à Chicago, davantage les gangs à prédominance raciale afro-américaine et hispanique. Les gangs caucasiens auraient des connexions plus périphériques avec la structure centrale alors que les gangs asiatiques seraient complètement isolés. Deuxièmement, les conflits plus importants entre les entités afro-américaines, soit le quart des interactions, auraient lieu au sein même du gang (conflit intra-gang) ou entre gangs de même affiliation (conflit intra-nation, donc intra-consortium). Ce modèle d'interactions intra-consortiums serait pratiquement absent des écrits scientifiques, selon l'auteur, à l'exception des travaux de Decker et Curry (2002). Ces derniers ont étudié la vague importante d'homicides liés aux gangs commis durant les années 1990 à St-Louis. Certains de leurs résultats démontrent qu'une importante proportion des homicides a eu lieu entre des gangs appartenant à un même consortium plutôt qu'à des consortiums rivaux, tel que proposé par la conception populaire. En fait, $61,5 \%$ de toutes les victimes Crips ont été tuées par un autre membre Crips. Leur étude affaiblit l'image populaire des affrontements entre gangs rivaux.

La conception des conflits polarisée Crips versus Bloods demeure, bien que les travaux de Papachristos (2009) et Decker et Curry (2002) démontrent une structure du réseau social qui dévie du modèle traditionnel d'interactions attendu. Selon Papachristos (2009), ses résultats suggèrent que le rôle des coalitions serait davantage symbolique que fonctionnel. Ce type de résultats va à l'encontre de ce qui est attendu d'après les principes de psychologie sociale voulant qu'une structure sociale composée d'individus qui partagent des opinions et des croyances similaires tendront à évaluer de la même façon les situations, les gens ou les actions de manière à préserver l'équilibre relationnel de la structure globale (Heider, 1946). Nous croyons, tout comme Papachristos (2009), que les interactions intra-consortiums vont à l'encontre de ce principe et illustrent le déséquilibre structural dans l'ensemble des interactions entre gangs. Ce déséquilibre global pourrait être expliqué entre autres par trois facteurs: la désorganisation générale des gangs, le manque de cohésion et le pouvoir individuel d'action. Premièrement, 
Decker et Curry (2002) ont évoqué la désorganisation des gangs et de leurs quartiers à Chicago pour expliquer leur difficulté à organiser les homicides et les relations intra-coalitions. Cette désorganisation générale est également notée chez les gangs de San Diego (Decker et al., 1999), les gangs de St-Louis (Decker et Van Winkle, 1996), ainsi que les gangs de Milwaukee (Hagerdon, 1988) et de Seattle (Fleisher, 1995). Deuxièmement, le manque de cohésion influencerait la structure à deux niveaux: micro-systémique (inter-gang) et macro-systémique (inter-coalition). L'informalité de l'association des pairs (Trasher, 1927; Hagedorn, 1998) ainsi que la non-permanence et l'instabilité des formations affaiblissent la conception d'une structure sociale stable et cohésive. Le manque de cohésion influence la loyauté et la solidarité qui ne semblent plus contrôler le choix des victimes lorsqu'il est question de violence létale intra-gang ou intra-consortium, selon Decker et Curry (2002). Difficilement mesurable, mais toutefois non négligeable, le manque de cohésion (Klein et Maxson, 1994; Decker, 1996; Curry et Decker, 2003), ou «la cohésion spontanée» (Suttles, 1968: 176) du gang permet aux membres d'user d'initiative et d'innovation dans leurs comportements individuels, qui est en fait le troisième facteur rendant vulnérable l'équilibre structural.

Le gang est souvent l'unité de recherche étudiée dans la structure relationnelle intergang (voir Kennedy et al., 1997; Papachristos, 2006, 2009; McGloin, 2005). En mettant l'accent sur l'entité globale, nous dépossédons l'individu de son pouvoir d'action au sein de la structure. Le leadership, une caractéristique souvent clamée comme centrale à une organisation, est plutôt décrit comme étant volatil et diffus (Klein et Maxson, 2006). Le leadership serait davantage une propriété collective de certains membres du gang qu'un concept hiérarchique. Chacun agirait en fonction du leadership qu'il s'octroie (Klein, 1971). Tous deviennent donc maîtres de leurs actions, bien que celles-ci ne soient pas nécessairement tributaires de l'action collective du gang. Les inter_actions individuelles viennent, elles aussi, modeler l'équilibre de la structure globale des relations intergangs.

En somme, une structure sociale est délimitée par des entités qui partagent une identité commune. Elle est en constante mouvance en raison des nombreuses influences des facteurs contextuels, organisationnels et systémiques. Les modèles d'interactions traditionnels (rivalités inter-consortiums) illustrent les grandes tensions qui existent entre les différents gangs. Cependant, compte tenu de ce qui a été observé 
par Decker et Curry (2002) et Papachristos (2009), nous ne pouvons plus prétendre étudier les dynamiques relationnelles des gangs en appliquant uniquement une conceptualisation générale. Notre étude propose donc une méthodologie qui permet d'opérationnaliser et d'analyser les interactions entre les gangs sur un territoire donné. Il sera ainsi possible de constater dans quelle mesure une conception traditionnelle s'applique à la structure relationnelle des gangs montréalais.

\section{Source des données et méthodologie}

La plupart des études qui portent sur l'organisation et la structure des gangs se sont basées sur des données policières (entre autres Kennedy et al., 1997; Decker et Curry, 2002 ; McGloin, 2005 ; Klein et Maxson, 2006; Papachristos, 2009). La présente étude repose essentiellement sur les propos des membres de gangs et leurs interactions lors des entrevues de groupe. L'étude a été menée auprès de 20 contrevenants âgés de 14 à 18 ans $^{2}$ hébergés en garde ouverte, en vertu de la Loi sur le système de justice pénale pour les adolescents (LSJPA), au Centre jeunesse de Montréal-Institut universitaire. Le contact initial avec les participants potentiels a été établi par la responsable des unités de garde. Le principal critère de sélection était l'autorévélation quant à leur affiliation à un gang à Montréal. Nous avons ensuite rencontré les intéressés afin de leur présenter plus en détail l'étude et définir en quoi consistait leur participation à cette dernière. L'échantillon est composé de 20 participants appartenant à 15 gangs différents et identifiés sur le territoire montréalais. Notons que l'anonymat des participants a été préservé par l'emploi de noms fictifs; cependant les noms des gangs identifiés sont réels.

\section{Le choix d'une méthode: l'entrevue de groupe}

Cette recherche propose une méthodologie qui se démarque des méthodologies habituellement utilisées dans les études qui portent sur les

2. En fait, l'âge inférieur est fixé de sorte que le jeune puisse consentir par lui-même à sa participation à cette étude sans avoir besoin du consentement écrit de ses parents, tel qu'exigé par le Comité d'éthique du CJM-IU à ce moment, ce qui n'est plus le cas à présent. Pour ce qui est de la limite supérieure fixée, 18 ans, elle correspond à l'âge maximal lors de la commission du délit afin qu'un jeune puisse avoir recours aux services du Centre jeunesse de Montréal-Institut universitaire. 
gangs de rue, soit l'entrevue de groupe ${ }^{3}$. Quatre groupes composés de quatre participants ou plus (membres de gang de rue d'affiliation similaire et distincte) ont été rencontrés. Comme le mentionne Greenbaum (1988), les séances d'entrevues de groupe favorisent les interactions entre les participants. Bien que le contexte social de l'entrevue de groupe ne soit pas naturel, il reste qu'il permet d'observer les interactions du groupe dans un contexte social (Morgan, 1996). D'ailleurs, les données produites par ces interactions au cours des séances sont une riche source d'informations qui doivent être prises en compte dans l'analyse du matériel recueilli (Morgan, 1996; Madriz, 2000; Duggleby, 2005). L'essence même de cette recherche tient à la richesse des interactions entre les participants de même allégeance ou non, et à leur façon de confirmer, d'infirmer ou d'argumenter leurs propos. Chacun des groupes a été rencontré lors de deux séances d'entrevue de groupe d'une durée d'une heure et demie chacune.

\section{Première séance}

La première séance était composée de trois étapes. D’abord, les participants ont dû remplir individuellement la fiche signalétique et la fiche d'information sur leur gang. La fiche signalétique visait à obtenir un bref portrait des participants (âge, ethnie, niveau de scolarité complété). De plus, il leur a été demandé de décrire les motifs pour lesquels ils se retrouvaient en détention et quelles étaient leurs peines fixées par le tribunal de la jeunesse. Ensuite, la fiche d'information portant sur leur gang visait à familiariser le participant avec les thèmes abordés lors de

3. Diverses méthodologies ont été empruntées afin d'accéder au réseau social des gangs. Le recours aux données officielles liées aux homicides impliquant des gangs de rue a été priorisé dans les recherches de Cohen et Tita (1999), Deckey et Curry (2002), Kennedy, Braga et Piehl (1997), Papachristos (2009) et Rosenfeld, Bray et Egley (1999). Ce matériel d'analyse comporte un biais important quant à la classification même des incidents (sont-ils reliés à un gang ou non?). Sanchez-Jankowski (1991) a préféré rendre compte du réseau relationnel des gangs par l'observation in situ et/ou in actu. Outre le temps investi, les limites de ce choix méthodologique se résument à la difficulté liée à l'accès à la population et les implications d'une intégration dans un tel milieu. Les entrevues individuelles ont été favorisées par les chercheurs désirant connaître l'engagement des membres de gangs, notamment par Decker (1996), Decker et Curry (2000), Mourani $(2004,2006)$, Hamel et al. (1998) et Hébert, Hamel et Savoie (1997). Cette méthodologie favorise certainement l'expression du récit de vie du participant et permet de l'approfondir. Il reste que les entrevues individuelles peuvent parfois ne pas apporter toutes les informations ou les connaissances souhaitées lorsqu'il est question de rendre compte d'un processus dynamique, tel les relations sociales intergangs. 
l'entrevue de groupe. Une fois les documents remplis, la deuxième étape a commencé. Il a été demandé aux participants de repérer sur une carte de la ville de Montréal l'emplacement de leur gang. Tour à tour, ils ont délimité le territoire occupé par le gang auquel ils s'identifient. Pour ce faire, ils ont eu le choix d'utiliser un marqueur bleu ou rouge, s'il était important pour eux d'identifier l'appartenance de leur gang aux bannières Crips ou Bloods, ou le marqueur noir, si le gang n'était pas affilié à une de ces deux bannières. Il leur a été demandé ensuite d'identifier les gangs avoisinant le leur, ennemis et alliés. Il y a eu consensus de la part de l'ensemble du groupe pour localiser chacun des gangs sur la carte de Montréal. La troisième étape a consisté à créer une matrice d'interactions. Cette matrice est en fait un tableau où tous les gangs identifiés à l'étape précédente ont été notés. Par la suite, chaque gang a été mis en relation avec un autre gang ayant été identifié également à la deuxième étape (par exemple: gang 1 avec gang 2; gang 1 avec gang 3). Pour chaque pairage, que nous nommerons dyade dorénavant, les participants devaient, en groupe, déterminer s'il y avait absence de relations (0) ou s'il y avait présence de relations. Dans le dernier cas, s'il y avait présence de relations, ils devaient spécifier si ces relations étaient positives (1) ou négatives (-1) et dire pourquoi ils faisaient cette affirmation ${ }^{4}$. Une attention particulière a été accordée aux nuances apportées par les participants dans leurs explications et illustrations concernant la nature des interactions, puisqu'une telle dichotomisation ne rend pas nécessairement compte de la complexité des réseaux sociaux. C'est ainsi que la matrice du réseau social de chacun des groupes rencontrés a été construite.

\section{Seconde séance}

Comme il a été mentionné précédemment, une seconde séance a été prévue avec chacun des groupes rencontrés. Cette deuxième séance a permis aux participants d'évaluer la structure du réseau social à l'étude par l'analyse du sociogramme, soit l'illustration graphique de la matrice d'interaction formée lors de la première séance. D'un coup d'œil, il a été possible d'apprécier la nature des interactions des gangs ainsi que

4. Déterminer la présence et la nature des relations est nécessaire à l'élaboration de la matrice d'intéractions. Cette classification est motivée par le besoin de créer une matrice binomiale afin de dériver les mesures de centralité et de cohésion du réseau, tel que proposé dans la section d'analyses (Wasserman et Faust, 1994; Hanneman et Riddle, 2005). 
de soulever et commenter certaines particularités du réseau. Cette deuxième séance a servi à valider les informations obtenues lors de la première séance et à approfondir leur contenu. De plus, des questions thématiques avaient été prévues si toutefois ces thèmes n'étaient pas abordés spontanément lors des entrevues. Les principaux thèmes touchaient la nature des conflits, les nuances des dynamiques entre les membres à l'extérieur versus à l'intérieur des unités de réadaptation, la violence, ainsi que toutes les questions liées à la vengeance et à l'établissement d'une réputation individuelle versus celle du groupe.

\section{Échantillon}

Comme il a été mentionné, les 20 participants à cette étude appartenaient à 15 gangs actifs et distincts sur le territoire montréalais. Lors du processus d'entrevues de groupe, ils ont identifié 20 autres gangs, avoisinant les leurs et avec lesquels ils ont des interactions. L'échantillon total compte donc 35 entités (voir annexe). L'échantillon restreint fait référence aux 15 gangs, parmi les 35, dont au moins un membre a été rencontré lors des entrevues de groupe. Nous avons comparé l'échantillon restreint à l'échantillon total sur trois aspects: l'affiliation à un consortium, la composition ethnique prédominante des membres du gang et l'emplacement du territoire d'activité (voir tableau 1). En premier lieu, nous retrouvons dans l'échantillon total une distribution relativement similaire des gangs affiliés aux Crips, aux Bloods et à la catégorie nommée autre (qui n'a d'affiliation à aucune de ces deux bannières), alors qu'il n'en est pas de même pour l'échantillon restreint. Dans cette dernière catégorie, une plus petite proportion des gangs affirme ne pas s'identifier à l'une des deux grandes bannières afroaméricaines, soit $13 \%$ comparativement à $31 \%$ dans l'échantillon total 5 . En obtenant une plus faible proportion pour les gangs non affiliés aux bannières des Bloods ou des Crips, nous retrouvons donc une proportion légèrement plus importante de Bloods et de Crips, avec respectivement $40 \%$ et $47 \%$ dans l'échantillon restreint comparativement à $31 \%$ et $37 \%$ dans l'échantillon global. En deuxième lieu, les gangs afro-canadiens sont représentés dans une proportion similaire dans

5. Cette faible proportion pourrait être expliquée par des risques d'arrestation moins élevés pour les membres de gangs non identifiés aux Crips ou aux Bloods. Ce sont des gangs qui sont moins exposés, donc moins connus et moins visibles (leurs délits suivent également cette logique). 
l'échantillon restreint et global. Nous observons également une faible proportion pour l'ensemble des autres races, bien que celles-ci soient représentées de façon relativement fidèle. En troisième lieu, en ce qui concerne les territoires occupés par les gangs représentés dans l'échantillon restreint, nous observons une situation similaire. Dans les deux cas, le secteur de l'Est est surreprésenté avec la majorité des 15 gangs s'y localisant.

\section{Stratégies d'analyse}

Nous présenterons dans un premier temps le portrait descriptif des entités à l'étude (voir tableau 1). Il sera donc possible de comparer les différences qualitatives et quantitatives entre les divers consortiums afin d'établir les prémisses des dynamiques relationnelles, c'est-à-dire de décrire «les forces en présence» sur le territoire montréalais. Dans un deuxième temps, il sera question de l'analyse de la structure relationnelle des gangs à l'étude. La fréquence des types de dynamique selon les différentes dyades d'affiliation sera présentée (voir tableau 2). Il sera ainsi possible de constater dans quelle mesure la conception traditionnelle s'applique à la structure montréalaise. Finalement, des mesures de centralité seront dérivées afin de déterminer les gangs étant les plus centraux au sein de la structure en fonction du nombre, de la nature et de l'orientation des relations. Le programme d'analyse de réseau Ucinet VI (Borgatti et al., 2002) a été utilisé pour réaliser ces analyses.

\section{Portrait descriptif des gangs montréalais à l'étude}

L'éclectisme et l'hétérogénéité caractérisent la structure de la population générale des gangs (Klein et Maxson, 2006). Nous nous sommes donc penchés dans un premier temps sur cette variabilité afin de décrire les entités du réseau étudié (tableau 1). Il est alors possible de saisir un premier portrait des entités présentes sur le territoire montréalais, soit les 35 gangs identifiés par les participants (échantillon global). L'échantillon restreint sera décrit de façon plus complète compte tenu de la quantité et de la qualité des informations obtenues par les 15 gangs représentés lors des entrevues de groupe. 
TABLEAU 1

Portrait descriptif des gangs montréalais à l'étude

\begin{tabular}{|l|c|c|c|}
\hline & Bloods & Crips & Autres \\
\hline $\begin{array}{l}\text { Échantillon restreint } \\
(\mathbf{N}=\mathbf{1 5})\end{array}$ & $\mathrm{N}=7$ & $\mathrm{~N}=6$ & $\mathrm{~N}=2$ \\
\hline $\begin{array}{l}\text { Échantillon global } \\
(\mathbf{N}=35)\end{array}$ & $(\mathrm{N}=11)$ & $(\mathrm{N}=13)$ & $(\mathrm{N}=11)$ \\
\hline \hline Territoire & & & \\
\hline Centre-Ville & 0 & 0 & $50 \%(45,5 \%)$ \\
\hline Est & $66,7 \%(72,7 \%)$ & $71,4 \%(76,9 \%)$ & $0(9,1 \%)$ \\
\hline Ouest & $33,3 \%(27,3 \%)$ & $28,6 \%(23,1 \%)$ & $50 \%(45,5 \%)$ \\
\hline Composition ethnique & & & $0(18,2 \%)$ \\
\hline Afro-Canadiens & $50 \%(54,5 \%)$ & $85,7 \%(92,3 \%)$ & $50 \%(18,2 \%)$ \\
\hline Asiatiques & $16,7 \%(18,2 \%)$ & 0 & $50 \%(18,2 \%)$ \\
\hline Caucasiens & $16,7 \%(9,1 \%)$ & 0 & $0(18,2 \%)$ \\
\hline Hispaniques & $16,7 \%(9,1 \%)$ & $14,3 \%(7,7 \%)$ & 16 ans \\
\hline Taille des gangs & 17 ans & 16 ans & 2004 \\
\hline Moyenne & 93 membres & 94 membres & 45 membres \\
\hline Médiane & 50 membres & 55 membres & - \\
\hline $\begin{array}{l}\text { Âge moyen des membres } \\
\text { rencontrés }\end{array}$ & 2000 & 1998 & \\
\hline Année de création & & & \\
\hline
\end{tabular}

Les 35 gangs identifiés par les répondants se distribuent de façon relativement équilibrée, sans la prédominance d'une bannière en particulier. On dénombre 11 gangs Crips pour 13 gangs Bloods. Nous sommes loin, par exemple, d'une prédominance Crips dans une proportion de 3 contre 1, telle que constatée aux États-Unis (Landre et al., 1997; Delaney, 2006). La proportion de gangs se retrouvant dans la catégorie de ceux ne s'identifiant à aucune des deux bannières connues demeure importante, avec un dénombrement de 11 gangs pour l'échantillon global (31\%) et 2 gangs pour l'échantillon restreint (13\%). Ce résultat nuance l'hypothèse initiale d'une distribution exclusivement Bloods et Crips sur le territoire montréalais. L'affiliation d'un gang à une bannière peut être tributaire de l'emplacement du gang sur le territoire montréalais. Le territoire désigné par les membres de gangs interviewés 
indique le lieu principal d'activité du gang et, pour certains, leur lieu de résidence. L'ensemble du territoire montréalais a été divisé grossièrement en trois sections afin de faciliter les analyses comparatives: le Centre-ville, l'Est et l'Ouest du secteur du Centre-ville ${ }^{6}$. Sur les 35 gangs identifiés, aucun gang du secteur Centre-ville n'est affilié à une des deux grandes bannières Bloods ou Crips. Pour ce qui est du secteur à l'Est, on retrouve exclusivement des gangs affiliés aux deux bannières. Quant à l'Ouest, il y a autant de gangs qui ne s'identifient pas à une bannière en particulier que de gangs s'y affiliant. Le territoire montréalais ne serait donc pas uniquement organisé selon la conception polarisée Crips versus Bloods appliquée à la distribution des affiliations. De plus, aucun résultat n'indique une surreprésentation d'une bannière en particulier dans les trois secteurs. En fait, il y aurait une symétrie en termes de proportion des gangs s'identifiant aux Bloods et aux Crips pour les secteurs à l'Ouest et à l'Est.

En ce qui concerne la composition ethnique des regroupements dans notre échantillon global, il se dégage des propos des participants que la bannière Bloods comprend des gangs dont une grande majorité des membres est d'origine asiatique, caucasienne et hispanique, ce qui la distingue de la bannière rivale Crips, qui ne compte que des gangs à prédominance afro-canadienne (92\%) ou hispanique $(8 \%)$. Selon ce qui est rapporté par les participants, les gangs à prédominance afrocanadienne représentent $18 \%$ des gangs non affiliés à une bannière, $55 \%$ des gangs affiliés aux Bloods comparativement à $92 \%$ des gangs affiliés aux Crips. Bien que nous ne puissions comparer le contexte montréalais au contexte étatsunien, ce résultat va dans le même sens que ce qu'avancent les recherches étatsuniennes stipulant que ce sont les Bloods qui ont historiquement accueilli des membres d'origine autre qu'afro-américaine (voir Delaney, 2006). De plus, nous observons qu'aucune prédominance raciale ne se démarque au sein des gangs qui ne s'identifient pas à l'une des deux grandes bannières. Selon les participants à cette étude, aucun gang de cette catégorie ne serait majoritairement composé de membres d'origine hispanique.

6. Il faut noter que l'Est comprend également tout le Nord-Est, et l'Ouest comprend tout le Sud-Ouest. Le secteur Est comprend Montréal-Nord, Villeray, St-Michel, ParcExtension, Pie-IX et St-Léonard. 


\section{Caractéristiques démographiques et descriptives des 15 gangs à l'étude}

La particularité des gangs montréalais à l'étude s'apprécie en fonction de l'estimation de leur taille moyenne, de l'âge moyen de leurs membres ainsi que de leur année de création (voir tableau 1). Bien que l'estimation de la taille des 15 gangs à l'étude varie d'une façon importante, allant de 8 membres à un extrême de 230 membres, aucune différence n'est notée entre les deux consortiums en ce qui a trait à la taille moyenne des gangs affiliés (environ 90 membres). L'étendue des intervalles estimés de l'âge moyen des membres du gang varie également: la plus petite valeur de la borne inférieure est de 12 ans et la plus grande valeur de la borne supérieure est de 40 ans. L'intervalle moyen estimé est de 15 à 27 ans. Bien que la borne supérieure soit un peu plus élevée, cet intervalle s'inscrit dans l'estimation proposée par Winfree, Backstrom et Mays (1994), soit l'intervalle 14-24 ans et l'intervalle proposé par Hamel, Fredette, Blais et Bertot (1998), soit 13-23 ans. À titre comparatif, la moyenne d'âge des 20 membres rencontrés lors de cette étude est de 16 ans, ce qui correspond à ce que nous retrouvons approximativement dans ces recherches. Aucune différence significative n'est notée entre les différentes bannières. L'année de création du gang n'était pas connue de tous et elle a été vaguement estimée. La moitié des membres de gangs rencontrés ayant fourni cette information estime que l'année de création daterait d'environ une dizaine d'années. Pour les dix autres, les gangs seraient nouvellement établis depuis une période qui varie entre un an et quatre ans. En nous attardant à la différenciation de cette caractéristique selon les bannières représentées, nous retrouvons une proportion similaire. En moyenne, les participants ont estimé que les gangs affiliés aux Crips sont en place sur le territoire montréalais depuis neuf ans, comparativement aux Bloods qui y seraient depuis sept ans. Les gangs qui ne sont pas affiliés à aucun de ces deux consortiums y seraient depuis trois ans.

Nous avons également demandé aux participants quelle était, selon eux, la force qui caractérise leur gang en termes d'activités criminelles. Ces éléments ont été regroupés en sphères particulières (marché des drogues, violence, autre type d'actes criminels, argent, structure interne de l'organisation) afin de catégoriser la distribution des réponses des participants. Puisque certains participants ont identifié plus d'une force, nous avons dans un premier temps classé les gangs selon ce critère, soit 
les gangs spécialistes versus les non-spécialistes. Les membres de 6 des 15 gangs n'ont identifié qu'une seule sphère d'expertise et se retrouvent donc dans la catégorie des spécialistes, tandis que les participants de 8 autres gangs considèrent avoir plus d'un champ d'activités. Deux principaux résultats ressortent de cette classification. Premièrement, tous les participants, à l'exception de deux membres appartenant respectivement aux gangs South Side et Dangerous Street, ont indiqué que la vente de drogues était l'une des forces principales de leur gang. Ce premier résultat va de pair avec ce qui est répertorié dans les travaux des chercheurs étatsuniens, notamment dans les sondages réalisés par le National Youth Gang Center et publiés par l'Office of Juvenile Justice and Delinquency Prevention (OJJDP). Le domaine d'expertise lié aux drogues forme une des catégories de la classification d'un gang spécialiste. Les gangs spécialistes dans le domaine des drogues étaient au nombre de quatre. Alors que les six autres gangs sont impliqués dans ce domaine sans pour autant le considérer comme l'unique force de leur gang. Deuxièmement, les participants de trois gangs ont identifié la violence comme une sphère dans laquelle ils ne sont pas des spécialistes. La violence accompagne donc leurs autres activités criminelles. Les membres du gang hispanique des $18^{\text {th }}$ ont mentionné que la violence était leur sphère d'expertise et consoliderait leur réputation dans le milieu.

L'organisation interne des gangs a été abordée aux questions directement liées à la stratification au sein du gang, soit à la présence ou non d'une hiérarchie. Bien que l'organisation des gangs ne soit pas très complexe et que leur fonctionnement varie d'un gang à l'autre (SanchezJankowski, 1991; Hébert et al., 1997; Klein et Maxson, 2006), plusieurs participants ont confirmé la présence de leaders dans la constitution interne du gang ${ }^{7}$. Certains ont fait mention d'une gradation des statuts à l'intérieur du gang, voire une stratification hiérarchique. John, membre du gang $B M F$, indique que pour son gang, il y a seulement «des vétérans et des rookies». Les répondants des gangs $A Y B, 47, B M F$, Blue Devils et un membre des $13^{\text {th }}$ abondent dans ce sens: ils signalent que dans leurs gangs il y a une organisation interne caractérisée par la présence de vétérans. Easy, membre des Blue Devils, ainsi que Crooked, membre des J.O.K.E.R.S., semblent du même avis en ce qui concerne la

7. Huit des vingt participants n'ont pas répondu à cette question puisqu'ils n'étaient pas au courant de la structure interne du gang auquel ils appartenaient. 
typologie de stratification des statuts au sein des gangs, telle la typologie présentée notamment par Yablonsky (1997) (voir aussi Shelden et al., 2001). Il était question des BG's (Baby Gangster), des Straights $G$, des G's (Gangster) et des OG's (Original Gangster, les vétérans). Crooked et Easy indiquent que dans leurs gangs respectifs, ils sont considérés comme des Straight G. Le répondant du gang $\mathrm{Ve} C r e w$ et un des membres rencontrés des $18^{\text {th }}$ soutiennent qu'il existe également une stratification hiérarchique dans leur gang, sans pouvoir spécifier davantage. Les participants membres du gang $L g$ Side, du gang $R T C$ et le plus jeune membre rencontré du gang $13^{\text {th }}$ ont précisé qu'il n'existe pas de hiérarchie particulière dans ces gangs. Certaines contradictions existent entre les différents participants appartenant à un même gang, notamment entre les membres des $18^{\text {th }}$ et des $13^{\text {th }}$. Il est possible qu'une partie de ces informations leur échappent en raison leur âge et leur statut de membre juvénile. Les participants plus âgés s'exprimaient davantage sur cette question comparativement aux plus jeunes. Tout compte fait, il y existe certainement une diversité de configurations possibles. Ceci nous porte à croire qu'il n'y a pas qu'une configuration privilégiée par l'ensemble des gangs à l'étude, d'où l'hétérogénéité des entités à l'étude et leur complexité.

Ce portrait descriptif et statique nous permet de constater que nous nous éloignons légèrement de la conception d'un territoire partagé uniquement entre les deux grandes coalitions Bloods et Crips. Le territoire montréalais accueillerait des gangs qui ne s'identifient pas à ces deux consortiums. Dans quelle mesure la structure relationnelle des gangs intègre donc ces différentes entités sur le territoire montréalais?

\section{Le réseau social des gangs montréalais à l'étude}

La structure du réseau social des gangs montréalais à l'étude a été recréée en établissant les interactions entre ces derniers, en 2007. La matrice du réseau est composée des 15 gangs auxquels appartiennent les 20 participants de cette étude et des 20 autres gangs identifiés par les participants. Seuls les gangs dont un membre a été rencontré ont été mis en interaction avec l'ensemble des gangs identifiés. La matrice est donc une table de 15 par 35 puisque nous ne pouvons pas présumer la réciprocité des relations entre les gangs dont aucun membre n'a été interviewé et les gangs dont un membre l'a été. Le sociogramme du réseau social des gangs est présenté à la figure 1 . 
F I G URE 1

Sociogramme

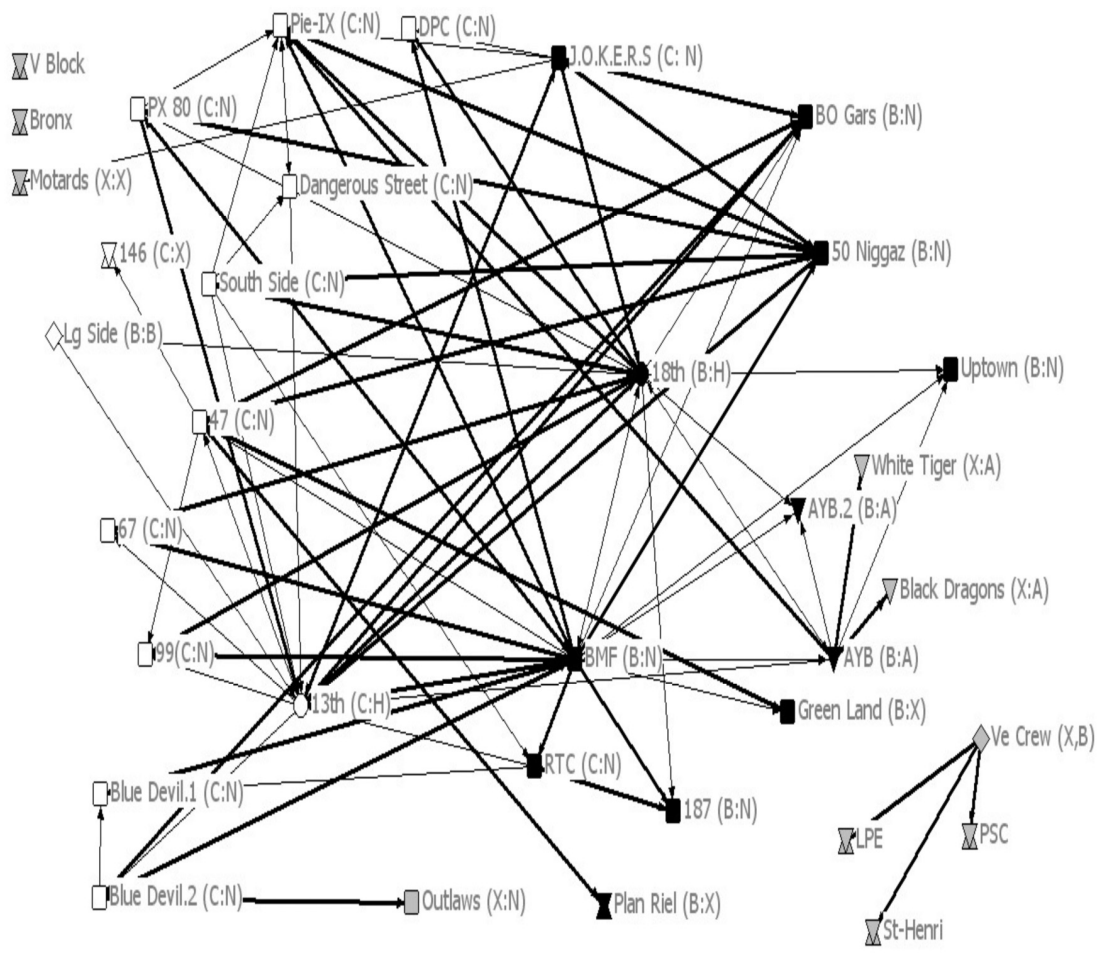

Note: La couleur blanche est utilisée pour désigner les gangs d'allégeance Crips, la couleur noire pour les gangs d'allégeance Bloods et la couleur grise pour les gangs qui ne s'identifient pas à l'une de ces deux bannières. Ensuite, le cercle représente un gang dont les membres sont majoritairement d'origine hispanique, le carré les gangs afro-canadiens, le losange les gangs caucasiens, le triangle les gangs asiatiques, et finalement les deux triangles superposés les gangs dont cette information n'était pas connue. Pour en faciliter la lecture, une parenthèse fournit une coordonnée qui résume ces informations. La première lettre fait référence à l'identification, si importance il y a, de la bannière Bloods (B), ou Crips (C), ou (X) autre. La deuxième lettre fait référence à l'identification de prédominance ethnique qui caractérise le gang: hispanique $(H)$, afro-canadienne $(N)$, caucasienne $(B)$, asiatique $(A)$ et $(X)$ aucun groupe ethnique spécifique.

\section{Organiser les dynamiques intergangs: la vision traditionnelle Crips versus Bloods}

La structure relationnelle à l'étude supporte en partie la vision traditionnelle et stéréotypée de l'organisation des dynamiques intergangs (voir tableau 2). Elle présente une majorité de relations négatives entre des gangs affiliés aux Crips et aux Bloods, soit $77 \%$ de l'ensemble des relations négatives. Les modèles de relations négatives concernent des 
entités qui s'identifient au même groupe ethnique (63\%). Les gangs afro-canadiens occupent $53 \%$ de cette proportion, ils sont donc majoritairement représentés. En ce qui concerne les relations positives, $88 \%$ d'entre elles sont entretenues entre des gangs de même bannière, tel qu'attendu par la conception traditionnelle. Les modèles de relations positives concernent des entités qui ne s'identifient pas au même groupe ethnique $(60 \%)$. Quelques modèles relationnels échappent à la règle et complexifient la compréhension de la structure. Il en sera question ultérieurement.

TA B LEA U 2

Alliances et conflits de la structure sociale selon les dyades d'affiliation

\begin{tabular}{|l|cc|cc|}
\hline & \multicolumn{3}{|c|}{ Alliances } & \multicolumn{2}{c|}{ Conflits } \\
\hline Dyades d'affiliation & \multicolumn{3}{|c|}{ Total } & \multicolumn{2}{c|}{ Total } \\
& $\mathrm{N}$ & $\%$ & $\mathrm{~N}$ & $\%$ \\
\hline Crips versus Crips & 18 & 45 & 2 & 4,7 \\
Crips versus Bloods & 5 & 12,5 & 33 & 76,7 \\
Bloods versus Bloods & 17 & 42,5 & 2 & 4,7 \\
Autres versus autres & 0 & 0 & 3 & 7 \\
Autres versus Crips & 0 & 0 & 1 & 2,3 \\
Autres versus Bloods & 0 & 0 & 2 & 4,7 \\
\hline Total & 40 & $100 \%$ & 43 & $100 \%$ \\
\hline
\end{tabular}

\section{Mesures d'analyse du réseau}

La densité, c'est-à-dire la proportion de tous les liens possibles qui sont effectivement présents (Degenne et Forsé, 1994; Wasserman et Faust, 1994), du réseau relationnel des gangs à l'étude (tous types de relations confondus) est de 0,112 , soit, $11 \%$ de tous les liens possibles entre les gangs identifiés sont présents. En différenciant l'ensemble des gangs selon les deux grandes coalitions Bloods et Crips, nous obtenons la densité pour l'ensemble des gangs affiliés aux Bloods, qui est de 0,020, c'est-à-dire que $2 \%$ de tous les liens entre les gangs Bloods sont présents. En ce qui concerne les gangs affiliés aux Crips, la densité de leur réseau est de 0,024 , soit $2,4 \%$ des liens entre gangs Crips du réseau social sont présents. Ce sont de faibles taux de densité typiques des réseaux des gangs étudiés par McGloin (2005), Klein et Maxson (2006) ainsi que Papachristos (2009). 
La centralité de degré pour chacun des gangs à l'étude a été mesurée afin de cerner les entités sociales qui se démarquent dans le réseau par une proportion plus importante de liens directs, positifs ou négatifs, qu'ils entretiennent avec le reste du réseau établi. La centralité de degré mesure en fait le niveau d'activité de l'acteur au sein du réseau (Wasserman et Faust, 1994). La centralité moyenne du réseau est d'environ sept liens pour l'ensemble des 35 gangs répertoriés. La structure globale a été décomposée en deux structures, c'est-à-dire une exclusivement composée de relations négatives et une autre exclusivement formée de relations positives. De plus, puisque les variables sont asymétriques, c'est-à-dire qu'il n'est pas assuré qu'une relation établie soit réciproque, nous avons divisé les mesures de centralité en out degree (destinateurs des connexions) et in degree (destinataires des connexions). Ce type de mesure de centralité quantifie dans quelle mesure un acteur émet des liens versus en reçoit à l'intérieur du réseau (Wasserman et Faust, 1994). Donc, la centralité out degree désigne le nombre de connexions qui provient d'un gang spécifique et qui est dirigé vers l'ensemble des gangs du réseau social. Subséquemment, cet élément s'oppose à la centralité in degree qui désigne la proportion des autres gangs du réseau social qui est connectée à ce gang spécifique. Le tableau 3 présente les gangs les plus centraux selon leur affiliation dans chacune des structures, soit exclusivement négative ou exclusivement positive.

\section{Les dynamiques attendues: Crips versus Bloods}

La conceptualisation traditionnelle des dynamiques conflictuelles intergangs répandue par les instances responsables de l'application de la loi ainsi que les médias suggère que la plupart de la violence intergang éclate entre les entités rivales et non pas entre les membres d'un même consortium (Jackson et McBride, 1985; Conly, 1993). Les différences d'allégeance seraient la source de conflits la plus véhiculée par la culture sous-jacente au phénomène et la plus médiatisée. Les bases de ce conflit seraient purement identitaires et clameraient l'appartenance à un groupe, à ses valeurs ainsi qu'à son territoire. En contrôlant les relations positives de la structure à l'étude, nous obtenons une structure exclusivement composée de dynamiques conflictuelles. Nous observons que le réseau conflictuel est dominé par la présence d'entités d'allégeance Crips, en termes d'émission, de destinateur des relations négatives (out degre). 
TABLEAU 3

Structure sociale clivée selon la nature et l'orientation des relations des 10 gangs les plus centraux

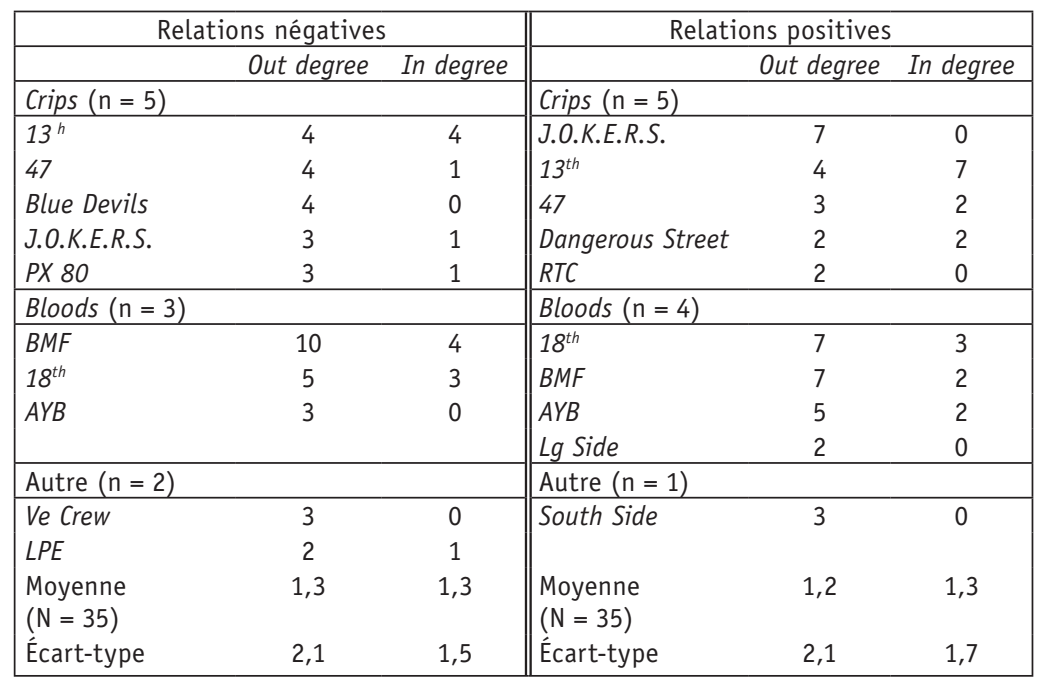

Bien que les deux premières positions des dix gangs les plus centraux soient détenues par des entités Bloods, nous constatons que cinq de ces gangs sont d'allégeance Crips, comparativement à trois gangs affiliés aux Bloods et deux seuls gangs d'autres affiliations. À l'inverse, les Crips sont également les entités les plus visées par les autres entités du réseau (in degree). Ces constats sont le reflet des propos de certains participants lorsqu'il était question du conflit purement associé aux différences d'allégeance. Une seule et même idée régnait: «Si tu n'es pas avec nous, tu es automatiquement contre nous» (Crooked, membre des J.O.K.E.R.S., gang Crips). Pour eux, il suffisait qu'un gang soit affilié à la bannière rivale de la leur pour conclure à la nature négative d'une relation entre les deux gangs. Cette affirmation valait même si ces gangs se retrouvaient sur un territoire opposé au leur et qu'ils avaient peu de risques de se croiser. Le simple fait de connaitre l'existence de ces gangs ainsi que la couleur marquant leur territoire suffisait pour prétendre qu'ils étaient des «ennemis jurés». À titre d'exemple, un membre des $B M F$ (gang Blood situé à Montréal-Nord et affilié au gang des Bo-Gars) confirme que toutes relations avec les membres des gangs associés aux Crips sont négatives, peu importe les circonstances. 
Négatif, parce qu'ils sont bleus [...], parce que c'est tout. Tout ce qui est bleu, on nettoie ça. [...] Pour mon secteur, yo, si c'est bleu, nettoie ça. Nous autres, on ne prend pas le temps de vérifier si tu es dans quel gang, ou si tu es loco [hispanique] ou si tu es vraiment un bleu, on nettoie ça. [...] Bleu, c'est tolérance zéro. (Membre du gang $B M F$ )

L'image du conflit «bleus versus rouges» est clairement illustrée par les propos de ce participant. Ce gang, les $B M F$, se taille une place centrale dans le réseau social en le dominant par le nombre d'interactions négatives qu'il émet (out degree de 10). Cependant, en s'attardant à la centralité in degree, nous constatons que la valeur de cette mesure chute à 4 . La différence du résultat est importante et soulève la non-réciprocité de certaines interactions. En fait, selon le participant, le gang des $B M F$ émettrait un nombre plus élevé d'interactions négatives vers d'autres entités du réseau que son propre gang serait visé par le nombre de relations émises par l'ensemble des autres gangs du même réseau. Le conflit n'est donc pas mutuel au niveau du groupe. La proportion des différentes orientations des relations identifiées n'est pas similaire, donc non équilibrée. Le conflit demeure réel selon la perspective individuelle du membre qui se définit comme une entité en conflit avec cet autre gang. Cependant, la réciprocité de cette perspective ne semble pas être démontrée par les propos des autres participants membres des gangs identifiés dans ces interactions négatives.

La structure à l'étude est alors influencée par l'agir individuel au sein du réseau, d'où la complexité des dynamiques et l'imprévisibilité du phénomène. Un membre du gang Crips J.O.K.E.R.S. illustre par ses propos l'inconsistance des réactions des membres d'un même gang. Bien qu'une animosité soit confirmée entre deux entités, l'actualisation de celle-ci et le niveau de réaction dépendront de chaque individu:

Si tu débarques dans l'Ouest [il s'adresse au participant membre du gang Bloods $B M F]$, je te vois. Je te dis si je n'enlève pas ton doigt pour l'envoyer à tous tes amis là [...]. Lui, il peut être très calme et vendre du crack et moi je peux tout de même aller piquer lui, lui et lui [en pointant trois autres participants de diverses allégeances] parce que ça me tente, peu importe c'est qui. (Membre du gang J.O.K.E.R.S.)

L'imprévisibilité des gangs de rue réside en partie dans le pouvoir d'action de chacun de leurs membres en ce qui a trait à l'organisation des interactions sur le territoire.

En ce qui concerne la structure composée de relations exclusivement positives, nous observons une forte propension aux dyades de gangs 
appartenant aux mêmes bannières, tel qu'attendu par la conception populaire. Nous observons que $45 \%$ des relations positives sont entretenues entre des gangs d'allégeance Crips et $43 \%$ entre des gangs Bloods, soit des proportions similaires. Tel que réalisé pour le réseau exclusivement composé de relations négatives, la centralité in degree a été calculée afin de déterminer la proportion de relations positives dirigées à l'égard du gang versus la centralité out degree afin de préciser la proportion de relations positives que le gang émet. Les moyennes de l'ensemble des gangs à l'étude concernant l'orientation des relations sont similaires: 1,2 pour les relations positives émises par les gangs (out degree) versus 1,3 pour les relations positives dirigées vers les gangs (in degree). Le gang Crips des $13^{\text {th }}$ est le seul qui obtient une plus grande proportion de relations positives dirigées envers son gang comparativement aux relations positives que ce gang émet envers les autres entités à l'étude. Ce gang partage entre autres le quartier St-Michel dans l'est de Montréal, où il y a une forte concentration de gangs Crips. Nous constatons que les gangs moins connus comme South Side, RTC, $\mathrm{Lg}$ Side et Dangerous Street se hissent dans les palmarès des gangs les plus centraux. Ces regroupements semblent plus discrets sur la scène montréalaise et prioriseraient donc des relations plus positives en guise de stratégie relationnelle dans leur réseau social. Un membre du gang Blood $B M F$, parle de l'alliance qu'ils ont avec les Bo-Gars, autre gang Bloods et désigné comme leur source d'approvisionnement:

Tu fais des échanges. Tu échanges des rues, [tu] échanges de la drogue. Comme eux [les Bo-Gars] ont la coke et puis si eux qui sont là veulent de la coke, ils vont aller acheter la coke aux Bo-Gars puis ils vont la vendre par la suite. (Membre du gang $B M F$ )

Les dyades positives intra-consortiums sont le type de dynamique positive le plus prisé dans la structure à l'étude. Ces relations demeurent éphémères puisqu'elles évoluent au rythme des dynamiques de marché. Selon les participants, si les deux parties évaluaient favorablement leur situation financière une par rapport à l'autre, il y aurait moins de problèmes et une meilleure possibilité d'entente entre elles. La structure sociale se modèle également en fonction des contextes externes dans lesquels elle est plongée. Si les marchés et les conditions d'affaires sont favorables à tous et si le respect règne en ce qui concerne la délimitation physique des territoires et les attentes informelles intergangs, les alliances peuvent résister. 


\section{Conceptualisation complexifiée : les conflits et les alliances inattendus}

Tout comme la division du territoire montréalais, les alliances et les conflits ne suivraient pas exclusivement l'orientation prescrite par les postulats de la conception traditionnelle. Étant donné la présence de gangs qui ne s'identifient ni aux Crips et ni aux Bloods $(\mathrm{n}=11$ pour l'échantillon global et $\mathrm{n}=2$ pour l'échantillon restreint), nous obtenons des modèles relationnels différents de ceux prévus par la conception traditionnelle et dichotomisée. Notre méthodologie a permis de retracer ces discordances en se concentrant sur l'interprétation individuelle des membres quant à leur propre réseau social.

\section{Les conflits inattendus}

Selon la conception populaire, les relations négatives ne seraient entretenues qu'entre entités appartenant aux bannières rivales Crips et Bloods. La structure sociale étudiée présente quelques exceptions à ce postulat. En fait, les participants ont identifié, entre autres, quatre dyades relationnelles entre gangs d'une même bannière qui seraient négatives (tableau 2). De plus, ils ont répertorié six dyades négatives impliquant des gangs d'allégeance autre que Crips ou Bloods. Les discordances relationnelles au sein d'une même bannière sont généralement nourries par des motifs personnels qui poussent ainsi les individus à exercer leur liberté de choix et d'action. A priori, si un gang autre que le leur s'affiche avec la même couleur, il se doit de maintenir la réputation du consortium auquel il s'identifie: "S'ils rep la même couleur que notre gang, eh bien qu'ils la représentent bien. S'ils font mal leurs affaires, on va devoir les régler» (Dawggi, membre du gang asiatique Blood $A Y B)$. Ceci serait une première piste générale expliquant la présence de violence intra-consortium: la sauvegarde de la réputation de sa bannière est une règle informelle de conduite que tout gang se charge d'appliquer. Ces animosités subjectives assureraient l'uniformité et la cohérence des entités qui s'identifient à un même consortium. Outre ce système de contrôle de «la qualité des forces en présence» sur le territoire, les participants ont précisé d'autres facteurs expliquant la présence d'incohérences.

Le principal facteur explicatif avancé par les participants est le fait que les gangs hispaniques et asiatiques ne s'identifient pas à ce conflit 
réduit aux bleus versus les rouges, bien qu'ils y soient confrontés. Les cinq participants affiliés aux gangs hispaniques $\left(13^{\text {th }}[\mathrm{n}=2]\right.$ et $\left.18^{\text {th }}[\mathrm{n}=3]\right)$ et le participant qui appartient à un gang asiatique $(A Y B)$ sont plutôt impliqués dans des conflits intra-consortiums. En ce qui concerne les entités hispaniques, certaines incohérences quant à leur allégeance sont dénotées par les participants. Loko, membre du gang $13^{\text {th }}$, indique qu'il y a des membres des $18^{t h}$, généralement associés à la famille des Bloods, qui s'identifient au contraire à la famille des Crips. À sa connaissance, la situation contraire ne s'applique pas au $13^{\text {th }}$, c'està-dire qu'aucun membre des $13^{\text {th }}$ ne s'identifie à la bannière des Bloods. John, membre du gang $B M F$ non hispanique, tend à le confirmer. Chele, membre des $18^{\text {th }}$, explique que la plupart des membres des $18^{\text {th }}$ se retrouvent à St-Léonard, un territoire marqué rouge. Mais nous trouvons également des membres des $18^{\text {th }}$ dans le quartier Pie-IX, un territoire associé au consortium Crips. Les conflits intergangs auxquels les gangs hispaniques sont confrontés sont davantage axés sur l'ethnicité des membres. À vrai dire, le conflit se situe plutôt entre les membres salvadoriens et les membres péruviens. «La majorité des membres hispaniques arborant le rouge sont d'origine salvadorienne», selon Crazy, membre des $18^{\text {th }}$. La structure relationnelle à l'étude présente deux dyades conflictuelles non attendues (Crips contre Crips) impliquant une entité hispanique. Le gang $13^{\text {th }}$ et le gang $P x-80$, tous deux sur le territoire de St-Michel, entretiendraient des liens négatifs. Le participant, membre de $P x-80$, précise que ce n'est pas un conflit purement personnel car d'autres membres de son gang ont également des problèmes avec les $13^{\text {th }}$. Le regroupement des $13^{\text {th }}$ ne tiendrait pas compte de la couleur bleue les unissant à cet autre gang. Un conflit à la base personnel, mais devenu une affaire de groupe, justifierait cette animosité. Le gang Crips des J.O.K.E.R.S. entretiendrait des liens négatifs avec le gang hispanique Crips des $13^{\text {th }}$. Le membre des J.O.K.E.R.S. mentionne qu'ils se parlent, mais il qualifierait tout de même leur connexion de nature négative. Il nous rappelle la philosophie de son gang: «Si tu n'es pas avec nous, tu es contre nous.» Pour ce qui est de Dawggi, membre du gang asiatique des $A Y B$, il affirme qu'il est impossible de comprendre les dynamiques conflictuelles qui définissent sa réalité en considérant uniquement la dichotomisation d'allégeance Crips versus Bloods. Ses ennemis portent le bandeau blanc ou le bandeau noir, respectivement les White Tiger et les Black Dragons, d'autres gangs asiatiques. Les sources de conflits ne sont pas liées aux affiliations Crips ou Bloods, bien 
que son gang soit établi sur un territoire marqué rouge. Il précise qu'il a des contacts avec certains Crips, des liens familiaux dont deux de ses frères, et qu' ils sont prêts à se rallier tous ensemble contre leurs ennemis communs (les White Tiger et les Black Dragons).

Les illustrations précédentes traduisent des incohérences dans le réseau social et portent à croire que les gangs qui ne sont pas concernés par la différenciation d'appartenance aux bannières Crips et Bloods ne seraient pas des gangs à prédominance raciale afro-canadienne. Cependant, cette même réalité est partagée par deux autres gangs Bloods majoritairement composés de membres afro-canadiens, soit le gang $B M F$ et les 50 Niggaz. Ces derniers partageraient non seulement des territoires associés aux Bloods, mais également une violente animosité. Tout compte fait, la conception polarisée serait, sans contredit, ébranlée. Non seulement par les exceptions observées sur la scène montréalaise, mais également par l'existence de gangs non affiliés à l'une des deux bannières, qui deviennent eux aussi des rivaux refusant de s'organiser autour de ce concept d'allégeance.

\section{Les alliances inattendues}

Les relations positives prédites selon la conception polarisée seraient présentes uniquement entre gangs qui appartiennent à un même consortium. La structure à l'étude présente cinq dyades relationnelles en désaccord avec cette conceptualisation. Des relations positives préexisteraient entre des entités qui appartiennent aux deux consortiums rivaux. Le motif majeur justifiant de telles dynamiques est lié à la spécificité des organisations hispaniques, «les affaires des locos», comme les participants les désignent. Les gangs hispaniques ne tiendraient pas compte des couleurs du gang pour laisser libre cours à leurs activités de fraternisation ou de collaboration d'affaires. Comme il a été mentionné auparavant, le gang asiatique $A Y B$ entretiendrait des liens d'alliance avec les Crips. Cette alliance serait premièrement justifiée par la présence de liens familiaux les unissant. Deuxièment, cette alliance serait motivée par un partage du territoire de Côte-des-Neiges, précisément par une réelle coexistence physique avec le gang hispanique des $13^{\text {th }}$. À titre de second exemple, le gang LG Side, gang Bloods, entretiendrait également de bons liens avec les hispaniques, tant les $13^{\text {th }}$ que les $18^{\text {th }}$. Le participant a affirmé qu'il avait des relations amicales avec des membres des deux clans et ce, sans qu'il y ait la moindre tension. 
Les alliances inattendues sont présentes dans l'organisation de la structure du réseau social à l'étude. Les gangs hispaniques ne sembleraient pas organiser leur réseau social de la même façon que le font les autres entités du réseau. Ils complexifieraient la compréhension que nous en avons en agissant contradictoirement à ce qu'il est prédit par la conception populaire. Il en serait de même pour le gang $B M F$, gang Bloods, qui a confirmé entretenir des liens d'affaires avec le gang 47, gang Crips. D’autres participants avaient considéré cette relation comme étant négative. Le membre du gang $B M F$ rétorque plutôt que ce gang est territorialement juxtaposé au leur et que cela favorise les relations d'affaires comme la vente de drogues ou d'armes. Tant que les deux entités tirent des avantages l'une de l'autre, le climat de tension n'existe pas.

Les conflits et les alliances inattendus dans la structure relationnelle soulignent la complexité du réseau social des gangs à l'étude et nous demandent une compréhension plus affinée des conflits intergangs, puisque chaque entité est concernée à des degrés différents.

\section{Conclusion}

Cette recherche avait pour but de déterminer dans quelle mesure les gangs montréalais s'organisent autour de la conception des alliances intra-consortiums et des rivalités inter-consortiums. Nous avons également proposé un cadre analytique unique afin d'étudier leurs dynamiques à partir du point de vue de 20 jeunes contrevenants hébergés en garde ouverte au Centre jeunesse de Montréal-Institut universitaire. L'entrevue de groupe a été la méthodologie priorisée et adaptée à l'objet d'étude afin que nous puissions examiner les entités identifiées et analyser la mise en interaction de celles-ci.

Selon les résultats de cette étude, le territoire montréalais se caractériserait par une majorité de gangs qui s'affilient aux consortiums Crips et Bloods. Par ailleurs, la présence de gangs qui ne s'identifient à aucune de ces deux bannières a été revélée et ce, particulièrement dans le secteur Centre-ville de Montréal. Les entités en place sur le territoire montréalais seraient similaires en termes de constitution interne (taille, âge moyen des membres, année de création). Sur le plan statique, il y aurait une symétrie «des forces en présence» sur le territoire. Sur le plan dynamique, la structure sociale est composée autant de relations négatives $(52 \%)$ que de relations positives (48\%). La densité de l'ensemble du réseau social est faible (11\%), soulignant ainsi le manque de 
cohésion en ce qui a trait à la structure générale des gangs (Klein, 1971; Klein et Maxson, 2006). Les alliances sont présentes dans $88 \%$ des dyades de gangs de même affiliation, alors que les conflits sont présents dans $77 \%$ des dyades de consortiums rivaux. Ceci constitue une représentation qui appuie majoritairement la conception traditionnelle d'une violence exclusivement actualisée entre membres d'une même bannière (Jackson et McBride, 1985; Conly, 1993). De plus, la majorité des conflits concernerait le même groupe ethnique (63\%). De cette proportion, $53 \%$ concerneraient les entités afro-canadiennes. À l'inverse, $60 \%$ des relations positives concerneraient des entités n'appartenant pas à un même groupe ethnique. Papachristos (2009) avait également dénoté une présence importante de conflits intra-raciaux, particulièrement parmi les entités afro-américaines. Ce type de résultat peut être expliqué par la densité de cette population ou par d'autres facteurs structuraux ou écologiques (Papachristos, 2009). Hipp, Tita et Boggess (2009) expliquent la violence intra-groupe en partie par la perspective de la désorganisation sociale générale. Les différences économiques diminueraient les contacts sociaux et cet isolement réduirait le contrôle social informel. Les tensions affaibliraient la qualité des liens sociaux, effriteraient la cohésion du groupe d'appartenance ainsi que du système de valeurs et de normes prôné. Par ailleurs, la coexistence entre entités appartenant à des groupes ethniques différents semble possible. La diversité et l'hétérogénéité caractérisent le territoire montréalais; Hipp et al. (2009) affirment qu'en territoire mixte, la violence entre les différents groupes ethniques n'est pas plus fréquente. Au contraire, la violence intra-groupe ethnique serait plus commune que la violence inter-groupe ethnique.

Outre les modèles relationnels qui s'inspirent de la conception traditionnelle, la structure à l'étude nous en présente d'autres s'y démarquant. Les exceptions concernent les deux consortiums. Tant les gangs Bloods que les Crips ont identifié des dynamiques conflictuelles intra-consortiums. Les conflits intra-consortiums (au sein d'une même bannière) représentent $9 \%$ des dynamiques négatives. Les travaux de Decker et Curry (2002) ainsi que de Papachristos (2009) avaient présenté ce même type d'exceptions. Contrairement à ces recherches, notre étude s'est intéressée également au réseau social positif des gangs. Les alliances interconsortiums (entre bannières dites rivales) sont représentées dans la structure à l'étude, avec $12,5 \%$ des dynamiques positives. Les structures des consortiums ont été créées afin de renforcer les alliances entre 
gangs qui s'identifient au même consortium afin de rivaliser avec le consortium adverse par solidarité et cohésion (Papachristos, 2009). La présence des modèles relationnels qui s'éloignent de la conceptualisation traditionnelle va donc à l'encontre à la nature même du phénomène des gangs et contredit les principes de l'équilibre structural. Les recherches sur les gangs ont souvent qualifié la structure des gangs comme souffrant de laxisme structurel (Klein, 1995; Decker et Curry, 2002). Les stratégies relationnelles reflèteraient cette désorganisation, à la fois de leur structure et celle des territoires et des quartiers qu'ils occupent. Les discordances remarquées démontreraient un effort d'adaptation et de coexistence de la part des gangs afin de s'assimiler à une structure en constant mouvement et influencée par les dynamiques des marchés illégaux.

Cet effort de coexistence a été perceptible tout au long des entrevues de groupe. Le design de cette recherche nous a permis d'interpréter ces résultats à la lumière des échanges entre les participants. Nous ne pouvons réduire la complexité du réseau relationnel des gangs à la conceptualisation simpliste d'un conflit Crips versus Bloods. Une conceptualisation restrictive est inadéquate pour prétendre comprendre le phénomène et y réagir. La complexité et le caractère désorganisé de la structure relationnelle reflètent un besoin d'organiser et de structurer les interactions sociales entre membres de gangs, peu importe le consortium. La solidarité, la cohésion et l'appartenance ne suffisent plus à freiner les conflits intra-consortiums.

Cette étude a tenté d'aborder le phénomène sous un nouvel angle de compréhension et d'analyse, en considérant les gangs comme des entités sociales en interactions, desquelles découlent des tendances en termes de stratégies relationnelles. Il serait maintenant pertinent de se dégager de ces grandes tendances et d'utiliser plus systématiquement cette méthode pour recueillir ce type d'informations, les traiter, les analyser et y réagir en appliquant ces nouvelles connaissances au contexte d'intervention. De plus, la perspective macro a été empruntée pour diriger cette étude; l'apport des individus demeure toutefois non négligeable et reste à explorer. D'ailleurs, croire et investir dans le potentiel qu'a chaque individu pour influer, à sa manière, sur l'ensemble du groupe et l'ensemble du réseau complexifie le caractère imprévisible du phénomène. Mais, en revanche, il nous permet également de garder espoir pour une issue plus positive de la situation montréalaise. 


\section{Références}

Borgatti, S. P., Everett, M. G., \& Freeman, L. C. (2002). UCINET 6 for Windows: Software for Social Network Analysis. Harvard: Analytic Technologies.

Chettleburrgh, M. C. (2007). Young thugs: Inside the dangerous world of Canadian Street Gangs. Toronto: Harper Collins Publishers Ltd.

Cohen, J., \& Tita, G. (1999). Diffusion in homicide: Exploring a general method for detecting spatial diffusion processes. Journal of Quantitative Criminology, 15, 451-493.

Conly, C. (1993). Street gangs; current knowledge and strategies. Washington, DC: National Institute of Justice.

Curry, G. D., \& Decker, S. H. (2003). Confronting Gangs: Crime and the Community (2d ed.). Los Angeles: Roxbury Publishing.

Decker, S. H. (1996). Collective and normative features of gang violence. Justice Quartely, 13, 243-264.

Decker, S. H., Bynum, T. S., \& Weisel, D. L. (1998). A tale of two cities: Gangs as organized crime groups, Justice Quartely, 15, 395-425.

Decker, S. H. \& Curry, D. G. (2002). Gangs, gang homicides, and gang loyalty: Organized crimes or disorganized criminals. Journal of Criminal Justice, 30, 343-352.

Decker, S. H., \& Van Winkle, B. (1994). "Slinging Dope": the role of gangs and gang members in drug sales. Justice Quarterly, 11, 583- 604.

Decker, S. H., \& Van Winkle, B. (1996). Life in the gangs: Family, friends and violence. Cambridge: Cambridge University Press.

Degenne, A., \& Forsé, M. (1994). Les réseaux sociaux. Une approche structurale en sociologie. Paris: Armand Colin.

Delaney, 2006. American Street Gangs. Upper Saddle River, NJ: Pearson Prentice Hall.

Duggleby, W. (2005). What About Focus Group Interaction Data? Qualitative Health Research, 15, 832- 839.

Felson, M. (2006). Crime and Nature. Thousand Oaks: Sage.

Fleisher, M. S. (1995). Beggars and Thieves: Lives of Urban Street Criminals. Madison: University of Winconsin Press.

Fleisher, M. S. (2005). Fieldwork research and social network analysis. Different methods creating complementary perspectives. Journal of Contemporary Criminal Justice, 21, 120-134.

Greenbaum T. L. (1988). The practical handbook and guide to focus group research. Lexington, Massachusetts/Toronto: Lexington Books.

Grennan, S., Britz, M. T., Rush, J., \& Barker, T. (2000). Gangs: An International Approach. New Jersey: Prentice Hall.

Hagedorn, J. (1988). People and Folks; Gangs, Crime, and the Underclass in a Rustbelt City. Chicago: Lake View.

Hamel, S., Fredette, C., Blais, M.-F., \& Bertot, J., en collaboration avec M.-M. Cousineau (1998). Jeunesse et gangs de rue (phase II) : résultats de la recherche- 
terrain et proposition d'un plan stratégique quinquennal. Rapport soumis au Service de police de la Communauté urbaine de Montréal. Montréal: Institut de recherche pour le développement social des jeunes.

Hanneman, R. A., \& Riddle, M. (2005). Introduction to social network methods. Riverside, CA: University of California, Riverside (published in digital form at http://faculty.ucr.edu/ hanneman/ )

Hébert, J., Hamel, S., \& Savoie, G.-J. (1997). Jeunesse et gangs de rue (phase I): revue de littérature, Rapport soumis au Service de police de la Communauté urbaine de Montréal. Montréal: Institut de recherche pour le développement social des jeunes.

Heider, F. (1946). Attitudes and Cognitive Organization. Journal of Psychology, 21, 107-112.

Hipp, J. R., Tita, G. E., \& et Boggess, L. N. (2009). Intergroup and intragroup violence: Is violent crime and expression of group conflict or social disorganization? Criminology, 47, 521-564.

Howell, J. C., Egley, A., \& Gleason, D. K. (2002). Modern day youth gangs. Washington, DC: Office of Juvenile Justice and Delinquency Prevention.

Jackson, R. K., \& McBride, W. D. (1985). Understanding street gangs. Sacramento, CA: Custom Publishing.

Kennedy, D. M., Braga, A. A., \& Piehl, A. M. (1997). The (Un)known universe: Mapping gangs and gang violence in Boston. In D. Weisburd \& T. McEwen (Eds.) Crime Mapping and Crime Prevention (p.219-262). New York: Criminal Justice Press.

Klein, M. W. (1971). Street gang and street workers. Englewood Cliffs, Nj: PrenticeHall, inc.

Klein, M. W. (1995). The American street gang: Its nature, prevalence, and control. New York: Oxford University Press.

Klein, M. W., \& Maxson, C. L. (1994). Gangs and cocaine trafficking. In Doris Mckenzie et Craig Uchida (eds.), Drugs and Crime: Evaluating public Policy Initiatives. Thousand Oaks, Calif.: Sage.

Klein, M. W., \& Maxson, C. L. (2006). Street Gang Patterns and policies. Oxford: Oxford University Press.

Landre, R., Miller, M., \& Porter, S. (1997). Gangs: A Handbook for Community Awareness. New York: Fact on File.

Madriz, E. (2000). Focus groups in feminist research. In N. Denzin \& Y. Lincoln (Eds.), Handbook of qualitative research ( $2^{\text {nd }}$ ed., 835-850). Thousand Oaks, CA: Sage.

Maxson, C. L. (1998). Gang members on the Move. Washington, D.C: Office of Juvenile Justice and Delinquency Prevention.

McGloin, J. (2005). Policy and intervention considerations of a network analysis of street gangs. Criminology $\sim \sim$ Public Policy. 4, 607-635.

Morgan, D. L. (1996). Focus groups. Annual Review of Sociology, 22, 129-152.

Morselli, C. (2009). Inside Criminal Network. New York: Springer. 
Mourani, M. (2004). Les bandes de jeunes de la région de Montréal et leurs liens avec les organisations criminelles. Mémoire de maîtrise, Université de Montréal, Montréal.

Mourani, M. (2006). La face cachée des gangs de rue. Montréal: Éditions de l'Homme.

Papachristos, A. V. (2006). Social network analysis and gang research: Theory and methods. In J. F. Short \& L. A. Hughes (Eds.) Studying Youth Gangs (99-116). New York: Alta Mira Press.

Papachristos, A. (2009). Murder by Structure: Dominance Relations and the Social Structure of Gang Homicide. American Journal of Sociology, 115, 74128.

Rosenfeld, R, Bray, T. M., \& Egley, A. (1999). Faciliting violence: a comparison of gang motivated, gang-affiliated and non gang youth homicides. Journal of Quantitative Criminology, 15, 495-516.

Sanchez-Jankowski, M. (1991). Islands in the street. Gangs and American urban society. Berkeley: University of California Press.

Shelden, R. G., Tracy, S. K., \& Brown, W. B. (2001). Youth gangs in American Society. Belmont (Ca): Wasworth Contempory.

Short, J. F. (1996). Gangs and adolescent violence. Boulder, CO: Center for the study and prevention of violence.

Short, J. F., \& Hughes, L. A. (2006). Studying Youth Gangs. New York: AltaMira Press.

Starbuck, D., Howell, J. C., \& Lindquist, D. J. (2001). Hybrids and other modern gangs (Juvenile Justice Bulletin, Youth Gang Series, NCJ No. 18916). Washington, DC: U.S. Department of Justice, Office of Juvenile Justice and Delinquency Prevention.

Suttles, G. D. (1968). The Social Order of the Slum: Ethnicity and Territory in the Inner City. Chicago: University of Chicago Press.

Thrasher, F. (1927). The gang. Chicago: University of Chicago Press.

Wasserman, S., \& Faust, K. (1994). Social Network Analysis: Methods and Applications. Cambridge: Cambridge University Press.

Winfree, L. T., Blackstrom, T. V., \& Mays, G. L. (1994). Social learning theory, self-reported delinquency, and youth gangs: A new twist on a general theory of crime and delinquency. Youth $\mathcal{O}$ Society, 26, 146-177.

Yablonsky, L. (1962). The Violent Gang. New York: MacMillan.

Yablonsky, L. (1997). Gangsters. New York: New York university press.

\section{Références électroniques}

National Youth Gang Center (2007). National Youth Gang Survey Analysis. [En ligne]. Consulté le 10 janvier 2007 de http://www.iir.com/nygc/nygsa/ 
ABSTRACT - This study proposes an analytical framework to study the network of street gangs in Montreal, including some affiliated to the two major American coalitions: Crips (blue) and Bloods (red). The objectives are two-fold: a) Describe the nature of the gangs themselves, emphasizing some of their core organizational features; b) Describe the relational dynamics within the network, once the gangs are allowed to interact (positively or negatively) with each other. We also want to understand in which ways the dynamics are orchestrated along the traditional perspective, that is, rivalries only occur between opposite consortiums. These objectives are fulfilled through an analysis of focus group interviews involving 20 youth gang members currently doing time in Centre jeunesse de Montreal-Institut universitaire. These youth gang members were affiliated to a diversity of gangs and coalitions. Together, they identified a total of 35 active gangs in Montreal. The resulting structure includes models of relationships expected by the traditional view, conflicts inter-coalitions (77\% of all negative relations $n=43$ ) and allies intra-coalition ( $87 \%$ of all positive relations $n=40$ ). Exceptions to the rule have been raised in our study and lead to a more nuanced understanding of Montreal's street gang network.

KEYWORDS • Gangs, internal structure, social network, intergangs interactions, alliances and conflicts.

RESUMEN - Este artículo propone un marco analítico para el estudio de la red de pandillas callejeras de Montreal, entre otras las que se identifican con dos de las grandes bandas de Estados Unidos, los Crips (azules) y los Bloods (rojos). El objetivo es doble: describir la naturaleza misma de las pandillas, principalmente sus características organizativas, y describir la dinámica relacional al interior de la red cuando las pandillas interactúan entre sí, de forma positiva o negativa. De manera específica, se investiga en qué medida las relaciones entre las pandillas se dan en términos polarizados Crips contra Bloods. Los datos se obtuvieron a partir de entrevistas de grupo realizadas en 2007 con 20 miembros de diferentes pandillas y coaliciones que estaban a cargo en ese momento del Centre jeunesse de Montreal-Institut universitaire. Los entrevistados identificaron 35 pandillas activas en Montreal en territorios específicos. La estructura derivada incluye modelos de relaciones de concepción tradicional, con conflictos inter coalición ( $77 \%$ de dinámicas negativas $n=43)$ y de alianzas intra coalición ( $87 \%$ del conjunto de dinámicas positivas $n=40)$. Las excepciones que el estudio encontró en esta dinámica confirman y matizan la estructura de relaciones encontrada en el estudio.

PALABRAS CLAVE - Pandillas, estructura interna, red social, interacciones entre pandillas, alianzas y conflictos. 
A N NEXE

Affiliation et territoire des gangs montréalais à l'étude

\begin{tabular}{|c|c|c|c|}
\hline & Bloods & Crips & Autre \\
\hline Centre-Ville & & & $\begin{array}{l}\text { Black Tigers } \\
\text { Outlaws } \\
\text { South Side } \\
\text { White Dragons } \\
\text { Motard }\end{array}$ \\
\hline Est & $\begin{array}{l}\text { Bo gars } \\
\text { BMF } \\
\text { Green Land } \\
\text { LG Side } \\
\text { Plan riel } \\
18^{\text {th }} \\
187\end{array}$ & $\begin{array}{l}\text { Dangerous Street } \\
\text { DPC } \\
\text { J.O.K.E.R.S. } \\
\text { Pie-IX } \\
\text { PX } 80 \\
50 \text { Niggaz } \\
13^{\text {th }} \\
67 \\
99 \\
146\end{array}$ & \\
\hline Ouest & $\begin{array}{l}\text { AYB } \\
\text { Uptown Posse }\end{array}$ & & $\begin{array}{l}\text { Bronx } \\
\text { LPE } \\
\text { PSC } \\
\text { St-Henri } \\
\text { V Block }\end{array}$ \\
\hline
\end{tabular}

Note: L'Est comprend également tout le Nord-Est et l'Ouest comprend tout le Sud-Ouest. Le secteur Est comprend Montréal-Nord, Villeray, St-Michel, Parc-Extension, Pie-IX et St-Léonard. 